\title{
Promoción de la salud mental de niños menores de 6 años
}

El proyecto de promoción de la salud mental de niños de 0 a 6 años ha formado parte de un plan de acción general de promoción de la salud mental, puesto en marcha por Salud Mental Europa (Federación Mundial de la S.M.) y financiando por la Comunidad Europea.

El proyecto ha tenido una duración de dos años. Comenzó en junio de 1997 y ha terminado en noviembre de 1999. Han participado los 15 países miembros de la Unión Europea y Noruega. Cada país ha estado representado por un coordinador nacional. La Asociación Española de Neuropsiquiatría ha asumido la representación española, formando un grupo de trabajo (Consuelo Escudero, Guillermo Larraz, Isabel Salvador, Ana Jimenez) con la colaboración del Ministerio de Sanidad (Rafael Peñalver).

Sus objetivos han sido:

- Desarrollar un concepto de promoción en salud mental en la edad preescolar

- Identificar, recoger y categorizar programas de promoción/prevención en todos los países europeos

- Organizar el intercambio de información entre todos los países europeos
- Desarrollar indicadores de actividades efectivas en la promoción de la salud mental de los niños

- Asesorar a la Comisión Europea sobre promoción en salud mental

La primera parte del proyecto (hasta febrero de 1998) ha consistido en la recogida y selección de programas de promoción/prevención de la S.M., de niños menores de 6 años, que se están realizando en Europa. Los criterios de calidad que han tenido que cumplir los proyectos son:

- Evidencia positiva sobre niveles de información cualitativa y cuantitativa

- Posibilidad de dirigirse a una amplia proporción de población con costes moderados

- Tener definidas claramente las metas a corto y medio plazo, y tener definidos los grupos a los que va dirigido

- Tener suficiente duración e intensidad para realizar los cambios oportunos en la salud mental, y en sus condiciones individuales y sociales 
INFORMES

- Ser desarrollado en periodos específicos

- Estar basado en el conocimiento científico sobre la temprana infancia y los determinantes sociales, psicológicos y neurobiológicos, más relevantes de la salud mental de los niños

- Haber realizado un proceso de evaluación

- Facilidad para que pueda ser repetido en cualquier contexto

Algunos de los problemas señalados por el grupo de expertos encargados de seleccionar los programas han sido: información insuficiente para valorar si algunos de los programas cumplían o no los criterios de calidad, otros tenían como objetivo secundario la prevención, siendo en realidad programas de intervención asistencial o bien de investigación, algunos iban dirigidos a niños mayores de 6 años, ausencia de un proceso de evaluación que ratificara la efectividad del programa, finalmente otros estaban todavía en periodo de prueba.

Se han seleccionado 195 programas llevados a la practica en diferentes contextos, sanitario, educativo, servicios sociales, etc., y realizados por diferentes organizaciones tanto estatales como privadas. Los proyectos españoles seleccionados han sido los siguientes:

Detección Precoz en situación de riesgo Departamento de Servicios Sociales de Alcázar de San Juan

Angel Parreño
Programa del niño sano

Gerencia de Atención Primaria, Instituto Nacional de la Salud de Baleares

Carmen Vidal

Protocolos de medicina preventiva en la edad pediátrica. Programa de atención del niño sano. Salud Mental infantil y juvenil Atención Psiquiátrica y Salud Mental del Servicio Catalán de la Salud Josep Ballester

Programa de Apoyo Escolar para la Protección de la Infancia.

Servicio de Renovación Pedagógica. Dirección General de Educación. Consejería de Educación y Cultura.

Comunidad de Madrid

Blanca Varona

Programa de psicoprofilaxis al embarazo y parto

Instituto Nacional de la Salud.

Area Sanitaria 10, Getafe

Elva Landeo

Detección Precoz de Trastornos Profundos del Desarrollo

Unidad de Salud Mental de Niños

y Adolescentes de Pamplona

$\mathrm{M}^{\mathrm{a}}$ Josefa Iribarren

Programa de Psicología Pediátrica en Atención Primaria

Ayuntamiento-Instituto Nacional

de la Salud de Baleares

Margarita Mullet

Unidad Funcional para la atención a la salud mental de la primera infancia (UFAPI)

Unidad de Salud Mental Sant Martí Nort. 
INFORMES

Instituto Catalán de la Salud

Jorge Tizón

Programa de Supervisión de la Salud Infantil

Dirección General de Salud Pública.

Consejería de Sanidad de la Comunidad

Valenciana

Francisco Bueno

Escuela de Padres y Madres "Ben Surats" para familias en situación de riesgo psicosocial

Asociación Instituto SPORA para la promoción comunitaria y la calidad de vida

Xisco Roman

\section{Prevención en la primera infancia}

Asociación de Psicoterapia y Prevención Infantil

Yolanda González

A lo largo de estos dos años se han realizado numerosas conferencias, reuniones y jornadas sobre promoción/prevención, a nivel europeo, para difundir los objetivos del proyecto, así como para sensibilizar a las Administraciones y Gobiernos sobre la necesidad de desarrollar programas preventivos en la primera infancia.

Desde la AEN hemos informado a todos los socios del inicio del proyecto, solicitando su colaboración. Todos los participantes han recibido los programas españoles, así como el Ministerio de Sanidad que ha colaborado en el desarrollo del proyecto. Hemos presentado el proyecto europeo en las XVI Jornadas Nacionales de la Asociación que se han celebrado en Palma de Mallorca del 18 al 20 de noviembre.
Se ha publicado un directorio, con todos los programas seleccionados, y las recomendaciones sobre promoción de la salud mental en niños menores de 6 años, adoptadas por la red europea de promoción de la S.M. Desde la AEN hemos publicado un diptico con estas recomendaciones.

El objetivo final del proyecto es difundir los programas seleccionados y promover la necesidad de realizar actividades de promoción en S.M. de niños, tanto en los medios profesionales, como ante las Administraciones competentes.

A lo largo de estos dos años hemos comprobado que los problemas para llevar a cabo programas de promoción, son generales en la mayor parte de los países europeos, a excepción de los países nórdicos, que son los que más actividades desarrollan en este campo en la actualidad.

El problema mas generalizado ha sido la falta de una evaluación cuantitativa y cualitativa de los programas, lo que dificulta la comprobación de la eficacia real en la promoción de S.M. y los cambios necesarios a medio y largo plazo para promover efectos saludables en la población.

En nuestro país se ha producido un abandono progresivo de las actividades de promoción, fundamentalmente en el ámbito sanitario, debido a la presión asistencial, por lo que seria necesario trabajar con las diferentes Administraciones para poner de relieve la necesidad de incrementar programas de prevención/promoción en S.M.

Para ello, desde la AEN, nos pondremos en contacto con el Ministerio de Sanidad para presentar oficialmente el directorio y las recomendaciones finales; así como con otras Administraciones de la red 
INFORMES

educativa, de servicios sociales, etc. Nos mantendremos también en contacto con las instituciones y organizaciones, que han enviado los proyectos españoles, para seguir el desarrollo de los programas.

Tenemos previsto informar a otras Asociaciones profesionales que podrían estar interesadas en el trabajo de promoción en S.M.

Salud Mental Europa tiene previsto continuar el trabajo sobre promoción en
S.M. y ha solicitado nuestro apoyo para desarrollar otro proyecto "Promoción de la S.M. de niños, adolescentes y jóvenes adultos", financiado por la Comisión Europea. En principio hemos contestado afirmativamente a la espera de que sea aprobado.

Hay una pagina web con toda la información disponible hasta el momento www.mhe-sme.org/enmhp 